\title{
Breaking the bias in microbiology
}

\author{
We present a specially commissioned set of articles to mark UN International Women's Day that highlight gender \\ inequalities that impact women in academia, clinical research and healthcare, and celebrate the achievements of \\ female microbiologists.
}

$\square$ ender equality is a human right that is essential for the achievement of sustainable development and has been named as goal 5 of the UN Sustainable Development Goals (SDGs). Although women's rights have received increasing attention over the past decade, a UN report published in 2018 stated that women and girls are overrepresented among those with limited access to clean water, food and education, and are more likely to experience domestic violence, carry the burden of unpaid care and household work, have fewer protections and rights by law, and experience a gender pay gap. Disparities by gender are highest in lowerand middle-income countries, but common challenges in all countries affect women and girls. On 8 March 2022, UN International Women's Day highlights a global need to \#BreakTheBias and achieve a gender-equal world. This day provides an opportunity to highlight issues and challenges that still impede women's equality, to identify ways to accelerate progress and to celebrate the achievements of women. To mark this occasion, we present a set of specially commissioned articles in a focus on women in microbiology.

One problem that is often aired but remains unsolved is a lack of women in decision-making roles within academia, industry and science policymaking. While the percentage of females studying science at school age is over $50 \%$ in the United Kingdom, and despite efforts to improve recruitment and retention of women in science, numbers of women dwindle as you progress through the academic and career trajectory to more senior positions. This problem is called the 'leaky pipeline' and is not restricted to the United Kingdom. Women represented less than $30 \%$ of researchers worldwide in 2016 according to the UNESCO Institute for Statistics and only $35 \%$ of research grants awarded by the National Institutes of Health (NIH) in 2020 were to women. Gender biases likely contribute to these trends. For example, a randomized double-blind study in 2012 found that science faculty were more likely to rate male applicants as more competent and hireable, and offer a higher starting salary, compared with an identical application with a female name (Moss-Racusin, C. A. et al. Proc. Natl Acad. Sci. USA 109, 16474-16479; 2012). Interestingly, the gender of the hiring faculty staff member did not impact these results, highlighting that there is an implicit bias regardless of gender or sex. Of note, a recent study found that male and female undergraduate students with a female mentor were more likely to report a favourable experience, indicating that hiring bias will affect all students (Moghe, S. et al. PLoS ONE 16, e0260646; 2021).

As well as improving representation of women in senior positions, social and political issues that affect women in everyday life must not be ignored. We present two World View articles that tackle these issues. First, those who decide to marry will likely make a decision on whether or not to take their partner's name. Bala Chaudhary, who works on mycorrhizal ecology, discusses in her World View article the negative impact this can have on a career in science as well as implications for family life, the role of race, and how effective mentoring can help. Second, reproductive rights of people with a uterus are being challenged across the world. As of 2021, there were 24 countries where abortion is illegal in all circumstances and another 37 countries where it is only legal for health reasons. Recently, Senate Bill 8, also known as the Texas Heartbeat Act, banned abortion once a heartbeat can be detected. Kelly Ramirez, a co-founder of 500 Women Scientists and an assistant professor of soil microbial ecology in Texas, discusses in her World View article the issue of reproductive justice and argues that academia must do more to support those most at risk. These are both issues that women have to consider and that are rarely, if ever, acknowledged by higher education institutions. If we are to ever achieve diversity and equality, we must acknowledge the threats women experience beyond the workplace.

Bias is also prevalent in healthcare and clinical research. The most well-known example of this is coronary heart disease. Although incidence of coronary heart disease is lower in women, they experience a higher mortality rate and worse prognosis than men. This is attributed to a bias in treatment and care, and gender-specific attributes, such as sex hormones and pregnancy complications. There are many understudied and underfunded conditions that predominantly affect people with vaginas that intersect with microbiology, such as menopause, bacterial vaginosis and some infectious diseases. In this focus, in a Comment article, Eileen Scully, who studies HIV, argues that we must consider sex and gender when designing clinical studies and that representation of women in clinical trials will improve diagnosis, treatment and care for diseases, such as HIV. In another Comment article, Kedest Mathewos and Agnes Binagwaho, who work in global health, call attention to the double burden experienced by women of colour during infectious-disease outbreaks, for example Ebola and COVID-19. Public health measures inadvertently impact maternal health outcomes, increase the risk of domestic violence against women and exposure to infection. One opinion common to both of these Comment articles is the importance of sex-disaggregated data to develop evidence-based and effective policies and interventions to positively impact women in microbiology.

While our understanding of the gut microbiota and the role it plays in health and disease continues to improve, the vaginal microbiota lags behind. In this focus, in a Review Article, Jacques Ravel and colleagues provide an overview of the latest research on the human vaginal microbiota and argue that there is an urgent need to develop models for mechanistic studies because current animal models have different vaginal communities and therefore are not optimal. In another Comment article, Pawel Łaniewski and Melissa Herbst-Kralovetz discuss the interplay between the vaginal microbiota and menopause, which affects half of the world's population but is underresearched and underfunded. If we are to achieve healthy ageing and longevity, menopause and reproductive health must receive more attention from funders and researchers alike.

In spite of these hurdles, women have been at the forefront of microbiology throughout history. From Jane Hinton, best 
known for her role in the development of Mueller-Hinton agar in 1941, to Elizabeth Lee Hazen and Rachel Fuller Brown who discovered the anti-fungal nystatin in the 1950s, and Fanny Hesse whose suggestion to use agar instead of gelatin in the late nineteenth century transformed the way we culture microorganisms. More recently, Nobel Prize-winners Jennifer Doudna and Emmanuelle Charpentier were rewarded for their ground-breaking work on the development of CRISPR-Cas9 genome editing tools that have revolutionized science. To celebrate the achievements of women in microbiology, in a series of Turning Points articles, we asked four women, Francine Ntoumi, Elizabeth Bukusi,
Margaret McFall-Ngai and Esperanza Martínez-Romero, to share their personal stories and recount the moments that have shaped their research careers to date. Themes running through their personal reflections echo challenges encountered by many women in science: misogyny, decisions on where to study and work, working with limited resources, and taking risks. Nevertheless, these women succeeded and helped those around them, whether that be by mentoring young female scientists, empowering their communities or by providing improved healthcare to women, and we hope that their stories will be an inspiration to men and women alike.
What can we do to stand up for gender equality and \#BreakTheBias? As scientists we can incorporate sex and gender in our research questions, and use disaggregated data; as mentors we can acknowledge the inequalities women face and create support systems that consider race, ethnicity, reproductive needs and parenting for example; and as allies we can educate ourselves on disparities and provide a platform to celebrate and advocate for women, and ultimately create the tangible change that is needed.

Published online: 4 March 2022 https://doi.org/10.1038/s41564-022-01086-z 